\title{
Evolution of graduate attribute assessment and continuous program improvement in the Faculty of Engineering at McGill University
}

\author{
Amber L. Saunders and Laurent B. Mydlarski \\ McGill University, Faculty of Engineering \\ amber.saunders@mcgill.ca laurent.mydlarski@mcgill.ca
}

\begin{abstract}
Herein, we summarize the development and implementation of processes to meet the CEAB's graduate attributes and continuous program improvement criteria at McGill University's Faculty of Engineering. This involved determining roles and responsibilities, developing tools for measuring graduate attributes, conducting pilot projects, and refining our process. Like other institutions, we have encountered obstacles: changes to $C E A B$ reporting requirements, resource constraints, resistance to change, and the challenge of finding a learning management system that is adaptable to our needs. We concluded that it was necessary to adopt processes that i) were initially rudimentary, but could then be scaled up, and ii) were flexible enough to respond to the changing reporting requirements. Pedagogical assistance provided by McGill University's Teaching and Learning Services was also beneficial in developing the new outcomes-based assessment procedures.
\end{abstract}

Keywords: accreditation, graduate attributes, continuous program improvement, outcomes-based assessment

\section{INTRODUCTION}

In this paper, we present a brief historical overview of activities aimed at assessing graduate attributes and implementing a process of continuous improvement for undergraduate programs in the Faculty of Engineering at McGill University. Our purpose is simply to share our own experiences and contribute to the ongoing conversation within the engineering education community as institutions grapple with meeting the accreditation criteria established by the Canadian Engineering Accreditation Board (CEAB). Our approach appropriate for our context - was built around one guiding principle: for meaningful, accurate and beneficial results to be obtained, processes and procedures must designed to maximize their adoption by the professoriate and minimize the disruptions to current assessment and grading practices, while concurrently meeting all CEAB reporting requirements.

The first step was to create the process, a three-year project that evolved from discussions within the Faculty of Engineering's Committee on Teaching and Learning into a more comprehensive, faculty-wide process with a newly formed Continuous Program Evaluation and Improvement working group to provide ongoing resources and support. This year, that process underwent significant revisions to make it more sustainable. The second step was to actually measure graduate attribute performance (undoubtedly the most difficult step). Some instructors measured graduate attribute performance beginning in January 2012 in a few courses using their own methods and tools. This was followed by full-scale implementation of a faculty-wide process in September 2013 using in-house tools, which was in turn followed by another pilot project in 2014-2015 using McGill's (commercially-purchased) learning management software. Our next steps will involve evaluating the graduate attributes assessment pilot project, implementing assessment for all courses as of September 2015, analyzing previous data in the context of course and program improvement, and determining more precisely the most appropriate types of assessment methods to use for each of the attributes.

In the remainder of this paper, we describe in further detail the history of graduate attribute assessment and continuous program improvement in the Faculty of Engineering at McGill University in §2. In §3, we present the current status of our implementation of this process. In $\S 4$, we discuss some of the issues and problems we have faced, whereas in $\S 5$, we discuss some of the positive outcomes we have encountered in our work. In the last section, we provide some conclusions related to the entire process and our experiences. 


\section{HISTORY OF GRADUATE ATTRIBUTE ASSESSMENT AND CONTINUOUS PROGRAM IMPROVEMENT AT MCGILL}

\subsection{Organizational Aspects}

At McGill, the Associate Dean (Academic Programs) is ultimately responsible for accreditation submissions and the ensuing visits of engineering undergraduate programs. The assessment of graduate attributes and the implementation of a program of continuous improvement processes therefore automatically fell under the portfolio of the aforementioned Associate Dean. Initially, responsibility for developing the process of graduate attribute measurement and continuous program improvement was assigned to the Faculty of Engineering Committee on Teaching and Learning (CTL), and responsibility for implementing these processes would later be delegated to the Faculty of Engineering Academic Committee, both chaired by the Associate Dean (Academic Programs). Part of the mandate of the CTL is to improve the quality of teaching and learning in the Faculty; the mandate of the Academic Committee is to review and approving academic policies and all course and program changes. Membership of CTL includes one representative from each academic unit (department), the director of the University's Teaching and Learning Services (TLS), one undergraduate and one graduate student, a faculty Teaching Award winner, and an administrative staff member. Membership of the Academic Committee includes, among others, the faculty members responsible for curriculum changes in their department (in most cases the departmental curriculum committee chairs).

After three years (2009-2012) of discussions and work on graduate attributes by the CTL, a new committee was created in 2013, the Continuous Program Evaluation and Improvement (CPEI) working group, chaired by the Associate Dean (Academic Programs). This committee was tasked with implementing and further developing the processes and procedures that had been developed by the CTL. Most of the members on this group are also the departmental representatives on the Academic Committee (department curriculum committee chairs); two departments have delegated responsibility for graduate attributes to another faculty member. Membership also includes a representative from TLS, an undergraduate student representative, and an administrative staff member. In 2014, an administrative staff (non-faculty) position was created to manage graduate attributes processes. This person was also given the responsibility of initiating graduate attributes measurement using our Learning Management System (LMS), in collaboration with the university's instructional technology unit.
The Faculty experienced numerous changes in leadership from 2009 to 2014. During this period, the Dean changed three times and the Associate Dean (Academic Programs) changed four times due to terms ending and modifications to / restructuring of the portfolios of the Associate Deans. Chairmanship of the CTL changed twice during the period in which it was responsible for developing graduate attributes process, and chairmanship of the CPEI working group also changed twice. Membership on the CTL changed substantially each year. This resulted in challenges in maintaining continuity, consistency, and especially momentum in both developing and implementing processes related to graduate attributes. Continuity and consistency were ultimately maintained through documentation, verbal passing on of knowledge, and through the presence of some faculty and administrative staff members during the entire period. We do feel that some momentum was unavoidably lost; as a result, we are not as far along in implementing our processes as we would have liked.

\subsection{Refinement of a Graduate Attributes Process and Procedures}

In 2013, an attempt was made to synthesize four years of work and discussions of the CTL and CPEI into an implementable process, which was captured in a procedures document. These procedures were (or were supposed to have been) implemented for the 2013-2014 academic year. We described our overall process for continual program evaluation and improvement as a continuous, repeated cycle with the following elements:

1) Curriculum mapping: Course content and assessment are mapped to attributes and competencies (indicators).

2) Assessment: Student performance is assessed for a sampling of students and data is collected and reported to department chairs.

3) Analysis and interpretation of data: Results are used to inform departments about program quality.

4) Validation of curriculum content: Departments determine whether the program provides students with opportunities to be introduced to / develop / apply competencies associated with the graduate attributes and whether the courses indeed incorporate activities associated with the graduate attributes designated.

5) Program improvement: Actions are taken to improve courses and programs.

CTL had also created two to five performance indicators (dubbed "competencies") for each graduate attribute, with four performance levels (Not demonstrated, Marginal, Meets expectations, and Exceeds expectations) and accompanying descriptors for each indicator. In theory, performance was to be assessed for each indicator, as required for $\mathrm{CEAB}$ accreditation reporting. However, 
before implementing the process an important decision was made to assess graduate attributes only, not graduate attribute indicators. This decision was made to enable us to focus on implementing the overall process as quickly as possible. Once the process was running smoothly, we would later have to scale up the assessment to include indicators, a level of complexity which was impossible to implement in the early stages.

2.2.1 Curriculum mapping. In 2010, CTL had developed a method for mapping the curriculum whereby the intensity or level of each graduate attribute in each course was indicated on a scale from 1 to 5 , where 1 indicated little to no (below 25\%) graduate attribute content and 5 indicated that most or all (about 76-100\%) of the course was dedicated to developing that graduate attribute. A graduate attribute that was at a level of 3 or higher was to be assessed. In 2014, CPEI decided to replace the 1-5 levels with the new graduate attribute codes required for the Course Information Sheets in the 2013 CEAB Questionnaire (the most recent version of the Questionnaire at that time): Introduced (I), Developed (D), Applied (A), any combination of these (ID, IA, DA, IDA), or not applicable (N/A). All graduate attributes except those with a code of Introduced (or N/A) were to be assessed. The new codes were used for the sake of long-term efficiency (if we had to report the CEAB codes anyway, why not use them for our curriculum maps?); however, in the short term they caused some grief - we had previously created Faculty-wide course outlines based on the 1-5 mapping, and thus a significant project now had to be re-done. Moreover, by early 2015, the CEAB had changed the codes again. One conclusion we can draw from this is that graduate attributes processes must be resilient; $\mathrm{CEAB}$ reporting requirements have been fluctuating rapidly over the past four years, and we should probably expect further changes.

2.2.2 Assessment and sampling. According to our procedures, four attributes would be assessed each year in all programs, such that each attribute would be assessed every three years. Departments were required to assess only a sampling of students, although they could assess all students if they wished. We used a hybrid approach: each year, a certain percentage of incoming students in each program would be selected at random, and these same students would be assessed for graduate attributes throughout their studies. The percentage of students to be assessed was established based on program enrolment; for example, programs with an incoming cohort of between 12 and 30 students had to select at least $40 \%$ for this sample; for programs with an incoming cohort of 101 to 200 students, the sample would be $25 \%$. These percentages were selected based on the University's
Course Evaluation Policy and corresponded to the thresholds required to ensure reliability of course evaluation data [1]. In addition to this sample of students per program, a sampling of students registered within each course had to be assessed (if that course was being used to assess graduate attributes in that particular year). These course samples would include the students selected for the program samples, with additional students in the course selected if needed to form the required percentage.

The problem with this approach was two-fold: First, it was generally felt that the administration involved in selecting program and course samples and assessing only the sample of students would require more time and effort than simply assessing all students. Second, it was doubtful that assessing a graduate attribute only every three years would provide reliable data that could be used for program improvement anytime soon, or ever (considering the frequency of curriculum changes). Therefore, for the 2014-2015 academic year, departments were encouraged to assess all students in every course. In the meantime, a pilot project using our LMS to assess attributes that was run simultaneously promised to make the sampling and cyclical approach eventually obsolete.

To aid in assessment, an Excel spreadsheet was developed that could be used to automatically calculate the grade for each graduate attribute based on the grade for each assessment tool (e.g., test, lab, report), the weight of the graduate attribute in that assessment, and the weight of that assessment in the final grade. For example, a final exam might include questions that mapped to the Knowledge Base and Problem Analysis attributes, with a weighting of 0.6 for Knowledge Base (KB) and 0.4 for Problem Analysis (PA). The graduate attribute scores for $\mathrm{KB}$ and $\mathrm{PA}$ for that assignment would simply be the overall grade for assignment multiplied by 0.6 and 0.4 respectively. A similar calculation was used to calculate the graduate attribute score for the course. We were aware of the inherent flaw in this calculation: graduate attributes were not being directly measured because the graduate attribute grade was based on an overall grade on the assessment tool that aggregated the graduate attribute performance. However, this calculation was used to make it easier for instructors, enabling us to focus on quickly implementing the overall reporting process.

\subsubsection{Reporting assessment results, analysis and interpretation of data. Another Excel spreadsheet was provided that instructors were to use to report graduate attributes assessment in their course to their department chairs. All spreadsheets were to be compiled by department chairs (or designated individual) and used to show graduate attribute performance across the program. This data was then to be used by departments to inform decisions for program improvement.}




\subsection{Pilot projects}

Before the procedures described above were implemented in 2013-2014, initial attempts were made to assess attributes in some courses. Three attributes were measured in five courses during the Winter 2012 term. Because no specific procedures were in place, instructors used their own methods to measure performance on attributes. Though results were not tracked systematically, some participating instructors who were also CTL members shared some aspects of their experience at that committee.

In 2014, a small team (instructional technology managers, Faculty administrative staff members and the Associate Dean (Academic Programs) was formed to work with project managers from the company providing our LMS to initiate graduate attributes measurement in a small number of courses in 2014-2015 using curriculum mapping and program outcomes software, recently purchased as part of the university's LMS. In addition to measuring and reporting graduate attribute performance in the pilot courses, the objectives of this pilot project were to develop an efficient and sustainable process for measuring graduate attribute performance using our LMS and to assess the resources required to sustain the process.

Between fifteen and twenty hours of meetings were required to gather information, determine and implement the graduate attributes structure in the LMS for our courses and programs, and set up gradebooks. By this point, it was clear that because assessment tools (reports, exams, projects, etc.) usually assess multiple graduate attribute indicators, each grade item (e.g., question or problem) would have to be mapped to the appropriate graduate attribute indicators, and grades would have to be recorded for each grade item within an assessment tool to obtain accurate measurement of graduate attribute performance. Although obviously requiring much more grading time, the course gradebook data could be used to capture graduate attribute performance. This method was used in three pilot courses in Fall 2014. The pilot project is still ongoing; at the time of this writing, we are currently in the process of gathering and reporting the data from the Fall 2014 term, and we will be gathering feedback from instructors concerning the feasibility of this procedure next month. If successful, this will be implemented in all courses and programs in 2015-2016.

\section{CURRENT STATUS}

Prior to the 2014/15 academic year, the departments within the Faculty of Engineering at McGill had been following the processes and procedures (described above in §2.2) to various degrees. Some departments had made effectively no progress, whereas others had acquired substantial amounts of data using the procedures in place. One unit went beyond the procedures, mapping each question or problem on each assessment to a graduate attribute, grading every question for every student in some courses. However, no attempt at continuous program improvement had been made, as a complete set of data had yet to be collected in this process that must span multiple years to accumulate data for all 12 graduate attributes.

Given i) the lack of uniform progress across all departments in the Faculty, and ii) the rapidly approaching accreditation visit to nine of the ten Engineering programs at McGill in 2016, the Continuous Program Evaluation and Improvement working group decided to modify its approach to one that would hopefully streamline the process of graduate attributes analysis and enable the acquisition of a complete graduate attributes data set by the end of the 2015/16 academic year. To achieve this goal, it was realized that a procedure had to be put in place which rendered the acquisition of quantitative measures (measures that can readily be correlated with test, assignment, laboratory, exam, etc. scores) of graduate attributes to be (relatively) easily performed by course instructors. It was also hoped that simplifying the process of graduate attribute data acquisition would allow more meaningful and beneficial data to be collected. Moreover, to accomplish this goal, it was deemed best to minimize the disruptions to current assessment and grading practices, while nevertheless ensuring that all CEAB reporting requirements were met.

This change in effect had the following consequences. Firstly, it was decided that all graduate attributes subject to quantitative measurements (i.e. Knowledge Base, Problem Analysis, etc., as opposed to Professionalism and Lifelong Learning, which were better assessed by qualitative measures) be evaluated in all courses for every student every year. This had the benefit of i) eliminating the need to determine which subset of students in a given courses students were to be tracked/evaluated, ii) did not risk "missing" some data in a given course / over a given year / for a specified set of students, given that all data was always recorded (even if not all data would necessarily always be used), and iii) rendering the process of evaluating graduate attributes something that became the norm, performed for every student, in every course, each year. It furthermore did not require that instructors be required to evaluate students twice: once using standard academic performance measures, and again using rubrics to evaluate the graduate attributes of individual students in their course activities. The Continuous Program Evaluation and Improvement working group deemed that the professoriate would be unlikely to welcome such a doubling of the evaluation of students, as it would add drastically to their workload.

The proposed procedure to evaluate all quantitative measures of graduate attributes in courses necessitated that instructors associate each individual question on a graded course activity with a graduate attribute. (In other 
words, different questions on an assignment / test / exam might evaluate different attributes.) This therefore required that all grades be recorded on a question-byquestion basis, so that the performance of students in achieving the various graduate attributes could be extracted at the end of the semester.

To further streamline this process and to minimize the additional workload on individual professors, it was decided that all instructors be required to enter their grades - on a question by question basis - in the McGill LMS. Moreover, instructors would then be required to provide the Faculty with an information sheet indicating the correspondence between each graded question and the graduate attribute best assessed by the given question. The perceived advantage of this approach was twofold. Firstly, it minimized the work relating to the analysis of graduate attributes by the individual instructors, by enabling the analysis of such data to be performed at the Faculty level, and hopefully by the LMS (see the next section). Secondly, it also had the benefit of providing data in a uniform format for all courses. (In prior years when departments were locally evaluating graduate attributes using an preformatted Excel spreadsheet circulated to all instructors, the resulting spreadsheets were not all completed in identical fashions, rendering compilation of the data difficult.) The revised approach is currently underway and the subject of a pilot project for the Winter 2015 semester.

We should also note that not all attributes are best measured by quantitative measures, as noted above. In these cases, more specific, individualized approaches are planned to evaluate the relevant attributes. However, the attributes that are best measured indirectly are less frequently occurring in our curricula, which will therefore allow this more work-intensive approach to be undertaken. The courses in which indirect, qualitative measures will be most used are i) two courses that all McGill Engineering students take: FACC 100 Introduction to the Engineering Profession (a first-year course) and FACC 400 Engineering Professional Practice (a final-year course), and ii) the final-year capstonedesign course present in all McGill Engineering curricula.

Lastly, as CEAB requires that indicators of graduate attributes be evaluated (and not the attributes themselves), our plan is to extend this approach to the graduate attribute indicators we have developed (discussed in section 2.2) in the 2015-2016 academic year.

\section{ISSUES AND PROBLEMS ENCOUNTERED}

One major issue we have encountered - one that must certainly be shared by most other Engineering programs in Canada - is the continuously changing requirements imposed by the Canadian Engineering Accreditation Board. The most recent version of the accreditation questionnaire [2] has substantial changes from the previous one, including the following:

(i) The addition of a definition of "Complex Engineering Problems," which may change how and what we evaluate with respect to certain graduate attributes.

(ii) Changes to the way in which curriculum maps must be completed (i.e. simply Introduced, Developed and Applied, no longer supporting combinations thereof). The consequence of this change is that we must redo our curriculum maps for a third time, since we had developed them prior to the "IDA" (and combinations thereof) approach being introduced.

(iii) Requiring in Table 3.1.1 that the Knowledge Base attribute be subdivided into "Mathematics," "Natural Sciences," "Fundamental Engineering Science" and "Specialized Engineering Science," which effectively increases the number of graduate attributes from 12 to 15 .

As we proceed with our revised approach, we have encountered other problems - some specific to our approach and others being more general. For example, instructors of certain courses offer some courses in a manner such that only the Knowledge Base and Problem Analysis attributes are evaluated, and neither is evaluated distinctly from the other. (This would be the case in a course in which all graded course activities are "typical" engineering problems.) In this situation, we have decided to advise instructors to classify such graded activities as Problem Analysis, which we deemed to be a higher-level attribute than Knowledge Base.

Another difficulty with our approach has been the ultimate analysis of the graduate attributes using our learning management system. We had hoped to be able to devise a method in which our LMS might automatically calculate students' performance in achieving the various graduate attributes, but it has fallen short. It appears that the next version of the LMS may have the required capability, but it will not be ready in time for our needs. Our "last resort" will be to export the question-byquestion grade data from our LMS to Excel and perform the analyses manually. This will still hopefully have the added benefit of having all data in an identical format.

With respect to the human side, we have been made aware of certain professors who have intentionally "underestimated" the degree of different attributes in their courses, in hopes of "escaping" the need to have them evaluated given our decision to not analyze attributes in a course that are mapped to the lowest level (Introduced). This is indicative of a problem in communicating our approach to the entire professoriate, as the procedure described in $\S 3$ is no different, and requires no further work, for courses in which the presence of an attribute is deemed strong than weak. We will aim to better communicate our approach by meeting with individual departments on this subject.

Lastly, but certainly not least, a major problem we have experienced is lack of personnel to undertake the 
large task of implementing a process of graduate attribute assessment and continuous program improvement. Even with a Continuous Program Evaluation and Improvement working group in place, the majority of the work has been undertaken by a few people within in Faculty. However, given that none of the people involved can contribute all their time to this daunting task - a task that must be done over and above the usual curriculum analysis associated with accreditation of engineering programs in Canada, in addition to their other responsibilities - the workload appears, at times, overwhelming.

\section{POSITIVE OUTCOMES}

\subsection{Collaborations}

One valuable outcome of our efforts to create processes for graduate attribute measurement has been a number of unplanned conversations and collaborations with units outside Engineering on the subject of program outcomes-based assessment. Membership on the Faculty CTL had always included a representative from the University's Teaching and Learning Services; now, their expertise and advice has become even more important. As a result of University-wide consultation in 2011 to select a new learning management system, it was discovered that not only Engineering but other faculties were interested in assessing program outcomes or were required by other accreditation bodies to do so. A small working group on curriculum mapping was created by the University's instructional technology unit to discuss this aspect in comparing potential learning management systems; membership included representatives from the faculties of Engineering, Education, Law, and Medicine, along with education technology managers. Those responsible for accreditation in the faculties of Engineering and Medicine have met several times to discuss program outcomes assessment and their respective accreditation reporting requirements in the search for a new learning management system. Recently, Teaching and Learning Services asked us to share what we had learned thus far with the Department of Food Sciences, which was seeking accreditation and was now required to report on program outcomes.

Outside the University, we also have appreciated and benefited from conversations and discussions (and perhaps some commiseration) with our counterparts in other universities, through participation in EGAD workshops, experiences shared during past CEEA conferences, and other informal conversations. These conversations may not have happened without the graduate attributes and continuous program improvement requirements. At the very least, it seems that engineering educators may be collaborating more about best practices in assessment, which by itself must result in some improvement in the quality of engineering education.

\subsection{Instructors' Reflection on the Presence of Graduate Attributes in Courses}

Another positive outcome of our process for graduate attribute measurement is the reflection required by instructors in evaluating graduate attributes in their own courses. Although instructors can easily and readily claim the presence of certain attributes in their respective courses when completing curriculum maps, our process ultimately obliges instructors to identify the assessment mechanisms for the various attributes that they deem present in their courses. For example, if an instructor claims that four attributes are present at a nonintroductory level in a given course they teach, then they must ultimately identify the ways in which these same four attributes are evaluated. Although we cannot claim this was done by design, our process benefits from compelling instructors to reflect on the attributes actually assessed in their course, versus those that they believe to be present and evaluated.

\section{CONCLUSIONS}

In our context, we found that it would be effectively impossible to implement a comprehensive and complete procedure for assessing graduate attributes in a single iteration. As a result, we initiated a scaled-down process, which could ultimately be scaled up to meet all CEAB requirements. Moreover, we found it essential to design a process that was flexible given that $\mathrm{CEAB}$ reporting requirements have been changing frequently, even though the CEAB criteria have not changed. We also noted that the willingness to adopt new outcomes-based assessment methods was not universal, and a certain resistance to change existed at times. Furthermore, our attempts to use our learning management system to aid in our evaluation of graduate attributes were not especially successful, although updates to our system may hold promise in the future. Lastly, we highlight the benefits of having experts in pedagogical assessment (from McGill's Teaching and Learning Services) involved in our discussions.

\section{References}

[1] Policy on official end-of-course evaluations. McGill University, 2012. Available as of April 20, 2015 from http://www.mcgill.ca/tls/teaching/course-evaluations/about/ policy.

[2] Engineers Canada, Questionnaire for Evaluation of an Engineering Program. Canadian Engineering Accreditation Board, Ottawa, ON, 29 Dec., 2014. Available as of April 20, 2015 from http://www.engineerscanada.ca/accreditationresources. 